Article

\title{
General Solutions for Descriptor Systems of Coupled Generalized Sylvester Matrix Fractional Differential Equations via Canonical Forms
}

\author{
Kanjanaporn Tansri and Pattrawut Chansangiam * \\ Department of Mathematics, Faculty of Science, King Mongkut's Institute of Technology Ladkrabang, \\ Bangkok 10520, Thailand; kanjanaporn.ts@gmail.com \\ * Correspondence: pattrawut.ch@kmitl.ac.th; Tel.: +66-9352-666-00
}

Received: 24 January 2020; Accepted: 8 February 2020; Published: 14 February 2020

check for updates

\begin{abstract}
We investigate a descriptor system of coupled generalized Sylvester matrix fractional differential equations in both non-homogeneous and homogeneous cases. All fractional derivatives considered here are taken in Caputo's sense. We explain a 4-step procedure to solve the descriptor system, consisting of vectorization, a matrix canonical form concerning ranks, and matrix partitioning. The procedure aims to reduce the descriptor system to a descriptor system of fractional differential equations. We also impose a condition on coefficient matrices, related to the symmetry of the solution for descriptor systems. It follows that an explicit form of its general solution is given in terms of matrix power series concerning Mittag-Leffler functions. The main system includes certain systems of coupled matrix/vector differential equations, and single matrix differential equations as special cases. In particular, we obtain an alternative procedure to solve linear continuous-time descriptor systems via a matrix canonical form.
\end{abstract}

Keywords: descriptor system; linear fractional differential equation; Caputo's derivative; Kronecker product; vector operator; Mittag-Leffler function

MSC: 15A16; 15A24; 15A69; 33E12

\section{Introduction}

A motivation of this work comes from the treatment of linear algebra and differential equations for control and system theory. Indeed, many physical processes can be formulated or at least approximated by a generalized state-space system or a linear continuous-time descriptor system, namely,

$$
\begin{aligned}
E x^{\prime}(t) & =A x(t)+B u(t), \\
y(t) & =C x(t)+D u(t),
\end{aligned}
$$

where $A, E \in M_{n, n}, B \in M_{n, m}, C \in M_{p, n}$, and $D \in M_{p, m}$ are constant matrices. Here, we denote the set of all $m$-by- $n$ real matrices by $M_{m, n}$, and we set $M_{n}=M_{n, n}$. The vector functions $x(t), u(t), y(t)$ represent the system state, the system input, and the system output, respectively. When $E$ is a singular matrix (i.e., rank $E<n$ ), the vector function $x(t)$ is called a generalized state vector or descriptor vector. Two simple examples of (1) are a planar pendulum modeled through Newton-Euler equations, and an electrical circuit [1] consisting of energy storage elements such as capacitors and inductors. A necessity and sufficiency condition for the system (1) to have a unique solution is the regularity, i.e., the matrix pencil $s E-A$ is nonsingular for some constants $s$ [2]. The regularity and two other attractive properties of the descriptor system, namely, the impulsive free and the asymptotical stability, turns out to be equivalent to the symmetry property of the solution of the associated Lyapunov equation; 
see, e.g., [3,4]. To solve a regular linear continuous-time system (1), many authors used an idea of transforming the generalized state vector $x(t)$ through a suitable matrix canonical form [5-7]. In this case, the system (1) is decomposed into a small number of simple subsystems, and the vector $x(t)$ is partitioned as a block-vector form. A coordinate form which is useful to verify specific properties of the system (e.g., controllability) is a singular value decomposition (SVD) [8]. See more information in [9-12].

On the other hand, there are renewal interests in linear matrix differential equations of Sylvester type. A homogeneous Sylvester matrix differential equation takes the form

$$
X^{\prime}(t)=A X(t)+X(t) B
$$

where $A, B \in M_{n}$ are constant matrices and $X(t) \in M_{n}$ is an unknown matrix function. An explicit form of the solution for this equation can be obtained through an equivalent scalar differential equation; see [13]. A simple non-homogeneous linear matrix differential equation takes the form

$$
X^{\prime}(t)=A X(t)+U(t)
$$

where $U(t) \in M_{n}$ is a given matrix function. In fact, its general solution is given by [14]

$$
X(t)=e^{t A} X(0)+\int_{0}^{t} e^{(t-s) A} U(s) d s .
$$

A general system of non-homogeneous coupled linear matrix differential equations takes the form

$$
\begin{aligned}
& X^{\prime}(t)=A X(t) B+C Y(t) D+U(t), \\
& Y^{\prime}(t)=E X(t) F+G Y(t) H+V(t) .
\end{aligned}
$$

Here, $A, B, C, D, E, F, G, H$ are constant matrices and $X(t), Y(t)$ are unknown matrix functions. Many authors [14-22] investigated certain special cases of this system on the coefficient matrices. A remarkable idea is to reduce the system of matrix differential equations to an equivalent vector differential equation via the vector operator or the diagonal-extraction operator. In this case, the general solution is given explicitly in terms of matrix power series concerning exponentials, hyperbolic functions, or Mittag-Leffler functions.

The above studies can be extended to matrix differential equations with a fractional derivative. Throughout this paper, we concern Caputo's fractional derivatives, and we use the superscript $(\alpha)$ to indicate the Caputo's derivative of order $\alpha$. A simple system of non-homogeneous linear matrix fractional differential equations takes the form

$$
X^{(\alpha)}(t)=A X(t)+U(t)
$$

where $\alpha \in(0,1]$. The solution is given as follows (see [23])

$$
X(t)=E_{\alpha}\left(t^{\alpha} A\right) X(0)+\int_{0}^{t}(t-s)^{\alpha-1} E_{\alpha}\left((t-s)^{\alpha} A\right) U(s) d s,
$$

where $E_{\alpha}$ is the Mittag-Leffler function with parameter $\alpha$. For the homogeneous case $U(t)=0$, the general solution is reduced to $X(t)=E_{\alpha}\left(t^{\alpha} A\right) X(0)$; see [23,24]. Another technique to solve these kinds of problems is to utilize the hybrid Jacobi and block pulse operational matrix of fractional integral operator; see [25]. A general system of non-homogeneous coupled linear matrix fractional differential equations takes the form

$$
\begin{aligned}
& X^{(\alpha)}(t)=A X(t) B+C Y(t) D+U(t) \\
& Y^{(\alpha)}(t)=E X(t) F+G Y(t) H+V(t)
\end{aligned}
$$


with unknown matrix functions $X(t)$ and $Y(t)$. The case $A C=C A$ and $B D=D B$ of the system was investigated in [17]. A linear matrix fractional descriptor system of the form

$$
A X^{(\alpha)}(t)=B X(t)+C U(t)
$$

was investigated in [19].

In the present work, we consider a descriptor system of coupled generalized Sylvester matrix fractional differential equations in a general form as follows:

$$
\begin{aligned}
& \sum_{i=1}^{k_{1}} P_{i} X^{(\alpha)}(t) R_{i}=\sum_{i=1}^{k_{2}} A_{i} X(t) B_{i}+\sum_{i=1}^{k_{3}} C_{i} Y(t) D_{i}+U(t), \\
& \sum_{i=1}^{k_{4}} Q_{i} Y^{(\alpha)}(t) S_{i}=\sum_{i=1}^{k_{5}} E_{i} X(t) F_{i}+\sum_{i=1}^{k_{6}} G_{i} Y(t) H_{i}+V(t),
\end{aligned}
$$

where $0<\alpha \leq 1$. To get an explicit form of the general solution, we apply the vector operator and Kronecker products to reduce the system to an equivalent system of coupled vector differential equations. The second step is to make a coordinate transformation to a simpler vector system with new variables. This is done by matrix partitioning according to a matrix canonical form concerning ranks. The third step is to solve the vector system obtained in the previous step. Here, we impose an assumption about the invertibility of a coefficient matrix in a similar way as in [19]. The last step is to transform the new variables to the original ones, so that an explicit formula of the general solution is obtained in terms of Mittag-Leffler matrix functions. After that, we investigate certain interesting special cases of the main system for both descriptor systems of coupled matrix equations, single descriptor matrix equations, and linear continuous-time descriptor (vector) system.

The organization for the rest of the paper is as follows. In Section 2, we recall some useful tools from linear algebra and fractional calculus for treating our problems. We explain how to solve the main system into four steps in Section 3, and an explicit form of the general solution is presented here. For the second step of the procedure, there are four cases of coefficient matrices, and they are named as subsections. In Section 4, we discuss some special cases of the main system concerning matrix descriptor systems. The results about linear continuous-time descriptor systems are extracted from the main system, and presented in Section 5. Finally, we conclude the paper in Section 6.

\section{Preliminaries}

In this section, we important tools that will be useful later in our investigation for solving system of linear matrix differential equations.

\subsection{The Kronecker Product and the Vector Operator}

Recall that the operator Vec $: M_{m, n} \rightarrow M_{m n, 1}$ transforms a matrix $A \in M_{m, n}$ to a column vector Vec $A$ by consecutive stacking the columns of $A$. The Kronecker product of $A=\left[a_{i j}\right] \in M_{m, n}$ and $B \in M_{p, q}$ is defined to be a block matrix $A \otimes B$ of order $m n \times n q$ whose $(i j)$ - th block is given by $a_{i j} B$.

Lemma 1 (e.g., [26]). For any matrices $A \in M_{m, n}, B \in M_{n, p}$ and $C \in M_{p, q}$, the following identity holds:

$$
\operatorname{Vec} A B C=\left(C^{T} \otimes A\right) \operatorname{Vec} B .
$$

\subsection{Mittag-Leffler Function}

The Mittag-Leffler function $E_{\alpha, \beta}$ is an entire complex-valued function depending on two parameters $\alpha, \beta>0$ defined by 


$$
E_{\alpha, \beta}(z)=\sum_{k=0}^{\infty} \frac{z^{k}}{\Gamma(\alpha k+\beta)}
$$

where $\Gamma$ is the Gamma function. In particular, we set $E_{\alpha}:=E_{\alpha, 1}$. For any $A \in M_{n, n}$, we define

$$
E_{\alpha, \beta}(A)=\sum_{k=0}^{\infty} \frac{1}{\Gamma(\alpha k+\beta)} A^{k}=I_{n}+\frac{1}{\Gamma(\alpha+\beta)} A+\frac{1}{\Gamma(2 \alpha+\beta)} A^{2}+\cdots
$$

See more information about Mittag-Leffler functions e.g., in [27]. The Mittag-Leffler function includes the following special cases:

$$
E_{1}(A)=e^{A}, \quad E_{2}\left(A^{2}\right)=\cosh A, \quad A E_{2,2}\left(A^{2}\right)=\left(E_{2,2}\left(A^{2}\right)\right) A=\sinh A .
$$

The following computations are used in later discussions.

Lemma 2 (e.g., $[15,21])$. For any $A, B \in M_{n, n}$, we have

$$
\begin{gathered}
E_{\alpha}\left(\left[\begin{array}{cc}
A & 0 \\
0 & B
\end{array}\right]\right)=\left[\begin{array}{cc}
E_{\alpha}(A) & 0 \\
0 & E_{\alpha}(B)
\end{array}\right], \\
E_{\alpha}\left(\left[\begin{array}{cc}
0 & A \\
B & 0
\end{array}\right]\right)=\left[\begin{array}{cc}
E_{2 \alpha}(A B) & \left(E_{2 \alpha, \alpha+1}(A B)\right) A \\
\left(E_{2 \alpha, \alpha+1}(B A)\right) B & E_{2 \alpha}(B A)
\end{array}\right] .
\end{gathered}
$$

2.3. Fractional Differential Calculus in Caputo's Sense

Denote the usual differential operator by $D$ and the floor function by $\lfloor\cdot\rfloor$. Let $f$ be a piecewise continuous function on $(0, \infty)$ which is integrable on any finite subinterval of $[0, \infty)$. For an integral-order case $\alpha \in \mathbb{N}$, we set $f^{(\alpha)}(t)=D^{n} f(t)$. For the fractional-order case $\alpha \in(0, \infty)-\mathbb{N}$, we define

$$
f^{(\alpha)}(t)=\frac{1}{\Gamma(\lfloor\alpha\rfloor-\alpha)} \int_{0}^{t} \frac{D^{\lfloor\alpha\rfloor} f(\tau)}{(t-\tau)^{\alpha-\lfloor\alpha\rfloor+1}} d \tau
$$

When $x(t)$ is a vector function, we denote by $x^{(\alpha)}(t)$ the vector function obtaining from $x(t)$ by coordinate differentiating.

Lemma 3 (e.g., [23]). The solution of the following non-homogeneous vector fractional differential equation of order $0<\alpha \leq 1$ :

$$
x^{(\alpha)}(t)=A x(t)+u(t), \quad x(0)=x_{0}
$$

where $A \in M_{n, n}$ is a given constant matrix, $u(t) \in M_{n, 1}$ is a given vector function, and $x(t) \in M_{n, 1}$ is an unknown vector, is given by

$$
x(t)=E_{\alpha}\left(t^{\alpha} A\right) x_{0}+\int_{0}^{t}(t-s)^{\alpha-1} E_{\alpha}\left((t-s)^{\alpha} A\right) u(s) d s .
$$

\section{Solving the Main System}

Our main problem to consider is as follows: 
Problem 1. Consider the following descriptor system of coupled generalized Sylvester matrix fractional differential equations of order $\alpha \in(0,1]$ :

$$
\begin{aligned}
& \sum_{i=1}^{k_{1}} P_{i} X^{(\alpha)}(t) R_{i}=\sum_{i=1}^{k_{2}} A_{i} X(t) B_{i}+\sum_{i=1}^{k_{3}} C_{i} Y(t) D_{i}+U(t), \\
& \sum_{i=1}^{k_{4}} Q_{i} Y^{(\alpha)}(t) S_{i}=\sum_{i=1}^{k_{5}} E_{i} X(t) F_{i}+\sum_{i=1}^{k_{6}} G_{i} Y(t) H_{i}+V(t),
\end{aligned}
$$

where all matrices are conformable. More precisely, let $a, b, m, n, p, q, r, s, t, k_{1}, k_{2}, k_{3}, k_{4}, k_{5}, k_{6}$ be natural numbers such that $a b=m q=n p=$ st. For each $i$, let $P_{i}, A_{i} \in M_{m, n}, R_{i}, B_{i} \in M_{p, q}, C_{i} \in M_{m, s}, D_{i} \in M_{t, q}$, $Q_{i}, G_{i} \in M_{a, s}, S_{i}, H_{i} \in M_{t, b}, E_{i} \in M_{a, n}$ and $F_{i} \in M_{p, b}$ be given constant matrices. Let $U(t) \in M_{m, q}$ and $V(t) \in M_{a, b}$ be given matrix functions. Find the unknown matrix functions $X(t) \in M_{n, p}$ and $Y(t) \in M_{s, t}$.

Problem 1 includes the works about matrix differential systems $[13,15,18-21]$. We shall proceed to find the general solution $X(t)$ and $Y(t)$ into four steps.

Step 1. Transform system (7) in unknowns $X(t), Y(t)$ to an equivalent system of vector differential equations in unknowns $\operatorname{Vec} X(t), \operatorname{Vec} Y(t)$.

For convenience, we denote

$$
\begin{gathered}
J=\sum_{i=1}^{k_{1}}\left(R_{i}^{T} \otimes P_{i}\right), \quad K=\sum_{i=1}^{k_{4}}\left(S_{i}^{T} \otimes Q_{i}\right), \quad M=\sum_{i=1}^{k_{2}}\left(B_{i}^{T} \otimes A_{i}\right), \\
N=\sum_{i=1}^{k_{3}}\left(D_{i}^{T} \otimes C_{i}\right), \quad L=\sum_{i=1}^{k_{5}}\left(F_{i}^{T} \otimes E_{i}\right), \quad T=\sum_{i=1}^{k_{6}}\left(H_{i}^{T} \otimes G_{i}\right) .
\end{gathered}
$$

Applying the vector operator and using Lemma 1, we have

$$
\begin{aligned}
\operatorname{Vec}\left(\sum_{i=1}^{k_{1}} P_{i} X^{(\alpha)}(t) R_{i}\right) & =\operatorname{Vec}\left(\sum_{i=1}^{k_{2}} A_{i} X(t) B_{i}+\sum_{i=1}^{k_{3}} C_{i} Y(t) D_{i}+U(t)\right), \\
\sum_{i=1}^{k_{1}}\left(R_{i}^{T} \otimes P_{i}\right) \operatorname{Vec} X^{(\alpha)}(t) & =\sum_{i=1}^{k_{2}}\left(B_{i}^{T} \otimes A_{i}\right) \operatorname{Vec} X(t)+\sum_{i=1}^{k_{3}}\left(D_{i}^{T} \otimes C_{i}\right) \operatorname{Vec} Y(t)+\operatorname{Vec} U(t), \\
J \operatorname{Vec} X^{(\alpha)}(t) & =M \operatorname{Vec} X(t)+N \operatorname{Vec} Y(t)+\operatorname{Vec} U(t) .
\end{aligned}
$$

Similarly, we get

$$
K \operatorname{Vec} Y^{(\alpha)}(t)=L \operatorname{Vec} X(t)+T \operatorname{Vec} Y(t)+\operatorname{Vec} V(t) .
$$

Step 2. Transform the vector systems (8) and (9) in unknowns $\operatorname{Vec} X(t), \operatorname{Vec} Y(t)$ to an equivalent system of vector differential equations in new unknowns $z_{1}(t), z_{2}(t)$.

We shall make matrix partitioning according to canonical forms of the coefficient matrices $J$ and $K$. We consider the case when $J$ and $K$ are nonzero matrices. Note that, if $J$ or $K$ is nonsingular, then we can multiply both sizes of equations with its inverse so that the equation can be reduced to a simple one. Thus, we divide our consideration into four cases as follows. 


\subsection{Case 1: J and K Are Singular}

We shall write $J$ and $K$ in a canonical form concerning ranks. Let us denote $l=\operatorname{rank} J$ and $r=\operatorname{rank} K$, so that $0<l<a b$ and $0<r<a b$. We can apply elementary row/column operations to obtain invertible matrices (being products of elementary matrices) $J_{1}, J_{2}, K_{1}, K_{2} \in M_{a b, a b}$ such that

$$
J=J_{1}^{-1}\left[\begin{array}{cc}
I_{l} & 0 \\
0 & 0
\end{array}\right] J_{2}^{-1}, \quad K=K_{1}^{-1}\left[\begin{array}{cc}
I_{r} & 0 \\
0 & 0
\end{array}\right] K_{2}^{-1}
$$

Now, we make matrix/vector partitioning as follows:

$$
\begin{aligned}
J_{1} & =\left[\begin{array}{l}
J_{11} \\
J_{21}
\end{array}\right], \quad J_{1} M J_{2}=\left[\begin{array}{ll}
M_{11} & M_{12} \\
M_{21} & M_{22}
\end{array}\right], \quad J_{1} N K_{2}=\left[\begin{array}{ll}
N_{11} & N_{12} \\
N_{21} & N_{22}
\end{array}\right], \\
K_{1} & =\left[\begin{array}{l}
K_{11} \\
K_{21}
\end{array}\right], \quad K_{1} L J_{2}=\left[\begin{array}{ll}
L_{11} & L_{12} \\
L_{21} & L_{22}
\end{array}\right],
\end{aligned}
$$

where all matrices are conformable, i.e., $J_{11} \in M_{l, a b}, K_{11} \in M_{r, a b}, J_{21} \in M_{a b-l, a b}, K_{21} \in M_{a b-r, a b}, M_{11} \in$ $M_{l, l}, N_{11} \in M_{l, r}, L_{11} \in M_{r, l}, T_{11} \in M_{r, r}, M_{12} \in M_{l, a b-l}, N_{12} \in M_{l, a b-r}, L_{12} \in M_{r, a b-l}, T_{12} \in M_{r, a b-r}$, $M_{21} \in M_{a b-l, l}, N_{21} \in M_{a b-l, r}, L_{21} \in M_{a b-r, l}, T_{21} \in M_{a b-r, r}, M_{22} \in M_{a b-l, a b-l}, N_{22} \in M_{a b-l, a b-r}$, $L_{22} \in M_{a b-r, a b-l}$ and $T_{22} \in M_{a b-r, a b-r}$. We make the following coordinate transformations:

$$
J_{2}^{-1} \operatorname{Vec} X(t)=\left[\begin{array}{l}
x_{1}(t) \\
x_{2}(t)
\end{array}\right], \quad K_{2}^{-1} \operatorname{Vec} Y(t)=\left[\begin{array}{l}
y_{1}(t) \\
y_{2}(t)
\end{array}\right]
$$

where $x_{1}(t) \in M_{l, 1}, y_{1}(t) \in M_{r, 1} x_{2}(t) \in M_{a b-l, 1}$, and $y_{2}(t) \in M_{a b-r, 1}$. We can respectively reduce Equations (8) and (9) using Equations (10)-(12) to the following:

$$
\begin{aligned}
& {\left[\begin{array}{ll}
I_{l} & 0 \\
0 & 0
\end{array}\right]\left[\begin{array}{l}
x_{1}^{(\alpha)}(t) \\
x_{2}^{(\alpha)}(t)
\end{array}\right]=\left[\begin{array}{ll}
M_{11} & M_{12} \\
M_{21} & M_{22}
\end{array}\right]\left[\begin{array}{l}
x_{1}(t) \\
x_{2}(t)
\end{array}\right]+\left[\begin{array}{ll}
N_{11} & N_{12} \\
N_{21} & N_{22}
\end{array}\right]\left[\begin{array}{l}
y_{1}(t) \\
y_{2}(t)
\end{array}\right]+\left[\begin{array}{l}
J_{11} \\
J_{21}
\end{array}\right] \text { Vec U }(t),} \\
& {\left[\begin{array}{ll}
I_{l} & 0 \\
0 & 0
\end{array}\right]\left[\begin{array}{l}
y_{1}^{(\alpha)}(t) \\
y_{2}^{(\alpha)}(t)
\end{array}\right]=\left[\begin{array}{ll}
L_{11} & L_{12} \\
L_{21} & L_{22}
\end{array}\right]\left[\begin{array}{l}
x_{1}(t) \\
x_{2}(t)
\end{array}\right]+\left[\begin{array}{ll}
T_{11} & T_{12} \\
T_{21} & T_{22}
\end{array}\right]\left[\begin{array}{l}
y_{1}(t) \\
y_{2}(t)
\end{array}\right]+\left[\begin{array}{l}
K_{11} \\
K_{21}
\end{array}\right] \operatorname{Vec} V(t) .}
\end{aligned}
$$

It follows that

$$
\begin{aligned}
x_{1}^{(\alpha)}(t) & =M_{11} x_{1}(t)+M_{12} x_{2}(t)+N_{11} y_{1}(t)+N_{12} y_{2}(t)+J_{11} \operatorname{Vec} U(t), \\
0 & =M_{21} x_{1}(t)+M_{22} x_{2}(t)+N_{21} y_{1}(t)+N_{22} y_{2}(t)+J_{21} \operatorname{Vec} U(t), \\
y_{1}^{(\alpha)}(t) & =L_{11} x_{1}(t)+L_{12} x_{2}(t)+T_{11} y_{1}(t)+T_{12} y_{2}(t)+K_{11} \operatorname{Vec} V(t), \\
0 & =L_{21} x_{1}(t)+L_{22} x_{2}(t)+T_{21} y_{1}(t)+T_{22} y_{2}(t)+K_{21} \operatorname{Vec} V(t) .
\end{aligned}
$$

Rearranging the system (13), we get the following equivalent system via block matrix multiplication:

$$
\left[\begin{array}{cccc}
I_{l} & 0 & 0 & 0 \\
0 & I_{r} & 0 & 0 \\
0 & 0 & 0 & 0 \\
0 & 0 & 0 & 0
\end{array}\right]\left[\begin{array}{l}
x_{1}^{(\alpha)}(t) \\
y_{1}^{(\alpha)}(t) \\
x_{2}^{(\alpha)}(t) \\
y_{2}^{(\alpha)}(t)
\end{array}\right]=\left[\begin{array}{cccc}
M_{11} & N_{11} & M_{12} & N_{12} \\
L_{11} & T_{11} & L_{12} & T_{12} \\
M_{21} & N_{21} & M_{22} & N_{22} \\
L_{21} & T_{21} & L_{22} & T_{22}
\end{array}\right]\left[\begin{array}{l}
x_{1}(t) \\
y_{1}(t) \\
x_{2}(t) \\
y_{2}(t)
\end{array}\right]+\left[\begin{array}{c}
J_{11} \operatorname{Vec} U(t) \\
K_{11} \operatorname{Vec} V(t) \\
J_{21} \operatorname{Vec} U(t) \\
K_{21} \operatorname{Vec} V(t)
\end{array}\right] .
$$

We transform $\left(x_{1}(t), x_{2}(t)\right)$ to $\left(z_{1}(t), z_{2}(t)\right)$ as follows:

$$
z_{1}(t)=\left[\begin{array}{l}
x_{1}(t) \\
y_{1}(t)
\end{array}\right], \quad z_{2}(t)=\left[\begin{array}{l}
x_{2}(t) \\
y_{2}(t)
\end{array}\right]
$$


so that $z_{1}(t) \in M_{l+r, 1}$ and $z_{2}(t) \in M_{2 a b-l+r, 1}$. For each $i, j \in\{1,2\}$, denote

$$
\Psi_{i j}=\left[\begin{array}{cc}
M_{i j} & N_{i j} \\
L_{i j} & T_{i j}
\end{array}\right], \quad W_{i}=\left[\begin{array}{cc}
J_{i 1} & 0 \\
0 & K_{i 1}
\end{array}\right], \quad w(t)=\left[\begin{array}{l}
\operatorname{Vec} U(t) \\
\operatorname{Vec} V(t)
\end{array}\right] .
$$

Using the coordinate transformations (14) and the notations (15), we get

$$
\left[\begin{array}{cc}
I_{2 l} & 0 \\
0 & 0
\end{array}\right]\left[\begin{array}{l}
z_{1}^{(\alpha)}(t) \\
z_{2}^{(\alpha)}(t)
\end{array}\right]=\left[\begin{array}{ll}
\Psi_{11} & \Psi_{12} \\
\Psi_{21} & \Psi_{22}
\end{array}\right]\left[\begin{array}{l}
z_{1}(t) \\
z_{2}(t)
\end{array}\right]+\left[\begin{array}{l}
W_{1} \\
W_{2}
\end{array}\right] w(t) .
$$

Now, we get the following system of coupled vector differential equations:

$$
\begin{aligned}
z_{1}^{(\alpha)}(t) & =\Psi_{11} z_{1}(t)+\Psi_{12} z_{2}(t)+W_{1} w(t), \\
0 & =\Psi_{21} z_{1}(t)+\Psi_{22} z_{2}(t)+W_{2} w(t) .
\end{aligned}
$$

Step 3. Solve the system consisting of (16) and (17) for $z_{1}(t), z_{2}(t)$.

In a similar manner to [19], we impose the following condition, which is related to the symmetry of the solution (e.g., [4]).

Assumption 1. Suppose that $\Psi_{22}$ is invertible.

We have from Equation (17) that

$$
z_{2}(t)=-\Psi_{22}^{-1} \Psi_{21} z_{1}(t)-\Psi_{22}^{-1} W_{2} w(t)
$$

Let us denote

$$
\mathcal{S}_{\Psi}=\Psi_{11}-\Psi_{12} \Psi_{22}^{-1} \Psi_{21} \quad \text { and } \quad \Gamma=W_{1}-\Psi_{12} \Psi_{22}^{-1} W_{2}
$$

Substituting the above equation into Equation (16), we obtain

$$
\begin{aligned}
z_{1}^{(\alpha)}(t) & =\Psi_{11} z_{1}(t)+\Psi_{12}\left(-\Psi_{22}^{-1} \Psi_{21} z_{1}(t)-\Psi_{22}^{-1} W_{2} w(t)\right)+W_{1} w(t) \\
& =\mathcal{S}_{\Psi} z_{1}(t)+\Gamma w(t) .
\end{aligned}
$$

Lemma 3 thus implies that

$$
z_{1}(t)=E_{\alpha}\left(t^{\alpha} \mathcal{S}_{\Psi}\right) z_{1}(0)+\int_{0}^{t}(t-\tau)^{\alpha-1} E_{\alpha}\left((t-\tau)^{\alpha} \mathcal{S}_{\Psi}\right)(\Gamma w(\tau)) d \tau
$$

Once we get $z_{1}(t)$, we can get $z_{2}(t)$ via Formula (18).

Step 4. Transform $z_{1}(t), z_{2}(t)$ back to $X(t), Y(t)$. We have

$$
\begin{aligned}
\operatorname{Vec} X(t) & =J_{2}\left[\begin{array}{l}
x_{1}(t) \\
x_{2}(t)
\end{array}\right] \\
& =J_{2}\left[\begin{array}{ll}
I_{a b} & 0
\end{array}\right]\left[\begin{array}{cccc}
I_{l} & 0 & 0 & 0 \\
0 & 0 & I_{a b-l} & 0 \\
0 & I_{r} & 0 & 0 \\
0 & 0 & 0 & I_{a b-r}
\end{array}\right]\left[\begin{array}{l}
z_{1}(t) \\
z_{2}(t)
\end{array}\right] \\
& =J_{2}\left[\begin{array}{cccc}
I_{l} & 0 & 0 & 0 \\
0 & 0 & I_{a b-l} & 0
\end{array}\right]\left[\begin{array}{l}
z_{1}(t) \\
z_{2}(t)
\end{array}\right] .
\end{aligned}
$$


To establish the solution $X(t)$ and $Y(t)$, we use the following transformations:

$$
\begin{aligned}
& \Phi_{1}: \mathbb{R}^{l+r} \times \mathbb{R}^{2 a b-(l+r)} \rightarrow M_{n, p}, \quad \Phi_{1}(u, v)=\operatorname{Vec}^{-1}\left(J_{2}\left[\begin{array}{cccc}
I_{l} & 0 & 0 & 0 \\
0 & 0 & I_{a b-l} & 0
\end{array}\right]\left[\begin{array}{l}
u \\
v
\end{array}\right]\right), \\
& \Phi_{2}: \mathbb{R}^{l+r} \times \mathbb{R}^{2 a b-(l+r)} \rightarrow M_{s, t}, \quad \Phi_{2}(u, v)=\operatorname{Vec}^{-1}\left(K_{2}\left[\begin{array}{cccc}
0 & I_{l} & 0 & 0 \\
0 & 0 & 0 & I_{a b-l}
\end{array}\right]\left[\begin{array}{l}
u \\
v
\end{array}\right]\right) .
\end{aligned}
$$

Thus, $X(t)=\Phi_{1}\left(z_{1}(t), z_{2}(t)\right)$ and, similarly, $Y(t)=\Phi_{2}\left(z_{1}(t), z_{2}(t)\right)$.

Theorem 1. The solution of Problem 1 under Assumption 1 is given by $X(t)=\Phi_{1}\left(z_{1}(t), z_{2}(t)\right)$ and $Y(t)=$ $\Phi_{2}\left(z_{1}(t), z_{2}(t)\right)$, where

$$
\begin{aligned}
& z_{1}(t)=E_{\alpha}\left(t^{\alpha} \mathcal{S}_{\Psi}\right) z_{1}(0)+\int_{0}^{t}(t-\tau)^{\alpha-1} E_{\alpha}\left((t-\tau)^{\alpha} \mathcal{S}_{\Psi}\right)(\Gamma w(\tau)) d \tau \\
& z_{2}(t)=-\Psi_{22}^{-1} \Psi_{21} z_{1}(t)-\Psi_{22}^{-1} W_{2} w(t) .
\end{aligned}
$$

In particular, for the homogeneous case $U(t)=V(t)=0$ of Problem 1, we have

$$
\begin{aligned}
& z_{1}(t)=E_{\alpha}\left(t^{\alpha} \mathcal{S}_{\Psi}\right) z_{1}(0), \\
& z_{2}(t)=-\Psi_{22}^{-1} \Psi_{21} z_{1}(t) .
\end{aligned}
$$

\subsection{Case 2: J and K Are Nonsingular}

From Equations (8) and (9), we have

$$
\begin{aligned}
& \operatorname{Vec} X^{(\alpha)}(t)=J^{-1} M \operatorname{Vec} X(t)+J^{-1} N \operatorname{Vec} Y(t)+J^{-1} \operatorname{Vec} U(t), \\
& \operatorname{Vec} Y^{(\alpha)}(t)=K^{-1} L \operatorname{Vec} X(t)+K^{-1} T \operatorname{Vec} Y(t)+K^{-1} \operatorname{Vec} V(t) .
\end{aligned}
$$

Thus, we can transform this system to the following equivalent system:

$$
\left[\begin{array}{c}
\operatorname{Vec} X^{(\alpha)}(t) \\
\operatorname{Vec} Y^{(\alpha)}(t)
\end{array}\right]=\left[\begin{array}{ll}
J^{-1} M & J^{-1} N \\
K^{-1} L & K^{-1} T
\end{array}\right]\left[\begin{array}{c}
\operatorname{Vec} X(t) \\
\operatorname{Vec} Y(t)
\end{array}\right]+\left[\begin{array}{l}
J^{-1} \operatorname{Vec} U(t) \\
K^{-1} \operatorname{Vec} V(t)
\end{array}\right]
$$

Lemma 3 now implies that

$$
\begin{aligned}
{\left[\begin{array}{l}
\operatorname{Vec} X(t) \\
\operatorname{Vec} Y(t)
\end{array}\right]=} & E_{\alpha}\left(t^{\alpha}\left[\begin{array}{cc}
J^{-1} M & J^{-1} N \\
K^{-1} L & K^{-1} T
\end{array}\right]\right)\left[\begin{array}{l}
\operatorname{Vec} X_{0}(t) \\
\operatorname{Vec} Y_{0}(t)
\end{array}\right] \\
& +\int_{0}^{t}(t-\tau)^{\alpha-1} E_{\alpha}\left((t-s)^{\alpha}\left[\begin{array}{ll}
J^{-1} M & J^{-1} N \\
K^{-1} L & K^{-1} T
\end{array}\right]\right)\left[\begin{array}{l}
J^{-1} \operatorname{Vec} U(\tau) \\
K^{-1} \operatorname{Vec} V(\tau)
\end{array}\right] d \tau
\end{aligned}
$$

Theorem 2. The solution of Problem 1 when $J$ and $K$ are nonsingular is given by

$$
X(t)=\operatorname{Vec}^{-1}\left(\left[\begin{array}{ll}
I_{a b} & 0
\end{array}\right]\left[\begin{array}{l}
\operatorname{Vec} X(t) \\
\operatorname{Vec} Y(t)
\end{array}\right]\right) \text { and } Y(t)=\operatorname{Vec}^{-1}\left(\left[\begin{array}{ll}
0 & I_{a b}
\end{array}\right]\left[\begin{array}{l}
\operatorname{Vec} X(t) \\
\operatorname{Vec} Y(t)
\end{array}\right]\right)
$$

where

$$
\begin{aligned}
{\left[\begin{array}{c}
\operatorname{Vec} X(t) \\
\operatorname{Vec} Y(t)
\end{array}\right]=} & E_{\alpha}\left(t^{\alpha}\left[\begin{array}{cc}
J^{-1} M & J^{-1} N \\
K^{-1} L & K^{-1} T
\end{array}\right]\right)\left[\begin{array}{l}
\operatorname{Vec} X_{0}(t) \\
\operatorname{Vec} Y_{0}(t)
\end{array}\right] \\
& +\int_{0}^{t}(t-\tau)^{\alpha-1} E_{\alpha}\left((t-\tau)^{\alpha}\left[\begin{array}{cc}
J^{-1} M & J^{-1} N \\
K^{-1} L & K^{-1} T
\end{array}\right]\right)\left[\begin{array}{c}
J^{-1} \operatorname{Vec} U(\tau) \\
K^{-1} \operatorname{Vec} V(\tau)
\end{array}\right] d \tau
\end{aligned}
$$


In particular, for the homogeneous case $U(t)=V(t)=0$ of Problem 1, we have

$$
\left[\begin{array}{c}
\operatorname{Vec} X(t) \\
\operatorname{Vec} Y(t)
\end{array}\right]=E_{\alpha}\left(t^{\alpha}\left[\begin{array}{cc}
J^{-1} M & J^{-1} N \\
K^{-1} L & K^{-1} T
\end{array}\right]\right)\left[\begin{array}{l}
\operatorname{Vec} X_{0}(t) \\
\operatorname{Vec} Y_{0}(t)
\end{array}\right] .
$$

3.3. Case 3: J Is Singular and K Is Nonsingular

Suppose $0<\operatorname{rank} J=l<a b$ and $\operatorname{rank} K=a b$. In a similar way to Case 1 and Case 2, we get the following matrix decompositions:

$$
J=J_{1}^{-1}\left[\begin{array}{cc}
I_{l} & 0 \\
0 & 0
\end{array}\right] J_{2}^{-1}, \quad K=K_{1}^{-1}\left[\begin{array}{cc}
I_{r} & 0 \\
0 & I_{a b-r}
\end{array}\right] K_{2}^{-1} .
$$

Then, Equations (8) and (9) can be transformed to the following equivalent system:

$$
\left[\begin{array}{cccc}
I_{l} & 0 & 0 & 0 \\
0 & I_{r} & 0 & 0 \\
0 & 0 & I_{a b-r} & 0 \\
0 & 0 & 0 & 0
\end{array}\right]\left[\begin{array}{l}
x_{1}^{(\alpha)}(t) \\
y_{1}^{(\alpha)}(t) \\
y_{2}^{(\alpha)}(t) \\
x_{2}^{(\alpha)}(t)
\end{array}\right]=\left[\begin{array}{cccc}
M_{11} & N_{11} & N_{12} & M_{12} \\
L_{11} & T_{11} & T_{12} & L_{12} \\
L_{21} & T_{21} & T_{22} & L_{22} \\
M_{21} & N_{21} & N_{22} & M_{22}
\end{array}\right]\left[\begin{array}{l}
x_{1}(t) \\
y_{1}(t) \\
y_{2}(t) \\
x_{2}(t)
\end{array}\right]+\left[\begin{array}{l}
J_{11} \operatorname{Vec} U(t) \\
K_{11} \operatorname{Vec} V(t) \\
K_{21} \operatorname{Vec} V(t) \\
J_{21} \operatorname{Vec} U(t)
\end{array}\right]
$$

Thus, the solution of Problem 1 under Assumption 1 is given by Formula (20), where

$$
\begin{aligned}
& \Psi_{11}=\left[\begin{array}{ccc}
M_{11} & N_{11} & N_{12} \\
L_{11} & T_{11} & T_{12} \\
L_{21} & T_{21} & T_{22}
\end{array}\right], \Psi_{12}=\left[\begin{array}{c}
M_{12} \\
L_{12} \\
L_{22}
\end{array}\right], \Psi_{21}=\left[\begin{array}{lll}
M_{21} & N_{21} & N_{22}
\end{array}\right], \Psi_{21}=M_{22}, \\
& W_{1}=\left[\begin{array}{ccc}
c_{11} & 0 & 0 \\
0 & K_{11} & 0 \\
0 & 0 & K_{21}
\end{array}\right], W_{2}=\left[\begin{array}{lll}
J_{21} & 0 & 0
\end{array}\right], w(t)=\left[\begin{array}{l}
\operatorname{Vec} U(t) \\
\operatorname{Vec} V(t) \\
\operatorname{Vec} V(t)
\end{array}\right], z_{1}(t)=\left[\begin{array}{l}
x_{1}(t) \\
y_{1}(t) \\
y_{2}(t)
\end{array}\right], z_{2}(t)=x_{2}(t) .
\end{aligned}
$$

\subsection{Case 4: J Is Nonsingular and K Is Singular}

Suppose $\operatorname{rank} J=a b$ and $0<\operatorname{rank} K=r<a b$. In a similar manner to Case 3, we get the following matrix decompositions:

$$
J=J_{1}^{-1}\left[\begin{array}{cc}
I_{l} & 0 \\
0 & I_{a b-l}
\end{array}\right] J_{2}^{-1}, \quad K=K_{1}^{-1}\left[\begin{array}{cc}
I_{r} & 0 \\
0 & 0
\end{array}\right] K_{2}^{-1} .
$$

Equations (8) and (9) are transformed to the following equivalent system:

$$
\left[\begin{array}{cccc}
I_{l} & 0 & 0 & 0 \\
0 & I_{a b-l} & 0 & 0 \\
0 & 0 & I_{r} & 0 \\
0 & 0 & 0 & 0
\end{array}\right]\left[\begin{array}{l}
x_{1}^{(\alpha)}(t) \\
x_{2}^{(\alpha)}(t) \\
y_{1}^{(\alpha)}(t) \\
y_{2}^{(\alpha)}(t)
\end{array}\right]=\left[\begin{array}{cccc}
M_{11} & M_{12} & N_{11} & N_{12} \\
M_{21} & M_{22} & N_{21} & N_{22} \\
L_{11} & L_{12} & T_{11} & T_{12} \\
L_{21} & L_{22} & T_{21} & T_{22}
\end{array}\right]\left[\begin{array}{l}
x_{1}(t) \\
x_{2}(t) \\
y_{1}(t) \\
y_{2}(t)
\end{array}\right]+\left[\begin{array}{l}
J_{11} \operatorname{Vec} U(t) \\
J_{21} \operatorname{Vec} U(t) \\
K_{11} \operatorname{Vec} V(t) \\
K_{21} \operatorname{Vec} V(t)
\end{array}\right]
$$


The solution of Problem 1 under Assumption 1 is given by Formula (20) with

$$
\begin{aligned}
& \Psi_{11}=\left[\begin{array}{ccc}
M_{11} & M_{12} & N_{11} \\
M_{21} & M_{22} & N_{21} \\
L_{11} & L_{12} & T_{11}
\end{array}\right], \Psi_{12}=\left[\begin{array}{c}
N_{12} \\
N_{22} \\
T_{12}
\end{array}\right], \Psi_{21}=\left[\begin{array}{lll}
L_{21} & L_{22} & T_{21}
\end{array}\right], \Psi_{21}=T_{22} \\
& W_{1}=\left[\begin{array}{ccc}
J_{11} & 0 & 0 \\
0 & J_{21} & 0 \\
0 & 0 & K_{11}
\end{array}\right], W_{2}=\left[\begin{array}{lll}
0 & 0 & K_{21}
\end{array}\right], w(t)=\left[\begin{array}{l}
\operatorname{Vec} U(t) \\
\operatorname{Vec} U(t) \\
\operatorname{Vec} V(t)
\end{array}\right], z_{1}(t)=\left[\begin{array}{l}
x_{1}(t) \\
x_{2}(t) \\
y_{1}(t)
\end{array}\right], z_{2}(t)=y_{2}(t) .
\end{aligned}
$$

\section{Descriptor Matrix Systems from Special Cases of the Main System}

In this section, we investigate certain interesting special cases of system (7) for both descriptor systems of coupled matrix equations and single descriptor matrix equations.

\subsection{Systems of Coupled Equations}

Corollary 1. Consider the descriptor system (7) when $\alpha=1$. From the notations and Assumption 1 in Section 3, we have

$$
z_{1}(t)=e^{t \mathcal{S}_{\Psi}} z_{1}(0)+\int_{0}^{t} e^{(t-\tau) \mathcal{S}_{\Psi}} \Gamma w(\tau) d \tau
$$

Proof. We just use the fact that $E_{1}(Z)=e^{Z}$ for any square matrix $Z$.

Corollary 2. Let $0<\alpha \leq 1$. Assume the notations and Assumption 1 in Section 3 for which $A_{i}=0, G_{i}=0$, $U(t)=0$ and $V(t)=0$, so that $M=T=0, M_{i j}=T_{i j}=0$ for each $i, j \in\{1,2\}$, and the invertibility of $\Psi_{22}$ is reduced to the invertibility of both $N_{22}$ and $L_{22}$. Then, the general solution of the system of coupled equations:

$$
\sum_{i=1}^{k_{1}} P_{i} X^{(\alpha)}(t) R_{i}=\sum_{i=1}^{k_{3}} C_{i} Y(t) D_{i}, \quad \sum_{i=1}^{k_{4}} Q_{i} Y^{(\alpha)}(t) S_{i}=\sum_{i=1}^{k_{5}} E_{i} X(t) F_{i}
$$

is given by

$$
X(t)=\operatorname{Vec}^{-1}\left(J_{2}\left[\begin{array}{l}
x_{1}(t) \\
x_{2}(t)
\end{array}\right]\right), \quad Y(t)=\operatorname{Vec}^{-1}\left(K_{2}\left[\begin{array}{l}
y_{1}(t) \\
y_{2}(t)
\end{array}\right]\right)
$$

where

$$
\begin{array}{ll}
x_{1}(t)=E_{2 \alpha}\left(t^{2 \alpha} \mathcal{S}_{N} \mathcal{S}_{L}\right) x_{1}(0)+t^{\alpha} E_{2 \alpha, \alpha+1}\left(t^{2 \alpha} \mathcal{S}_{N} \mathcal{S}_{L}\right) \mathcal{S}_{N} y_{1}(0), & x_{2}(t)=-L_{22}^{-1} L_{21} x_{1}(t) \\
y_{1}(t)=t^{\alpha} E_{2 \alpha, \alpha+1}\left(t^{2 \alpha} \mathcal{S}_{L} \mathcal{S}_{N}\right) \mathcal{S}_{L} x_{1}(0)+E_{2 \alpha}\left(t^{2 \alpha} \mathcal{S}_{L} \mathcal{S}_{N}\right) y_{1}(0), & y_{2}(t)=-N_{22}^{-1} N_{21} y_{1}(t)
\end{array}
$$

Here, $\mathcal{S}_{N}=N_{11}-N_{12} N_{22}^{-1} N_{21}$ and $\mathcal{S}_{L}=L_{11}-L_{12} L_{22}^{-1} L_{21}$.

Proof. From the notations and the assumption, we have

$$
\begin{aligned}
\mathcal{S}_{\Psi} & =\left[\begin{array}{cc}
0 & N_{11} \\
L_{11} & 0
\end{array}\right]-\left[\begin{array}{cc}
0 & N_{12} \\
L_{12} & 0
\end{array}\right]\left[\begin{array}{cc}
0 & L_{22}^{-1} \\
N_{22}^{-1} & 0
\end{array}\right]\left[\begin{array}{cc}
0 & N_{21} \\
L_{21} & 0
\end{array}\right] \\
& =\left[\begin{array}{cc}
0 & N_{11}-N_{12} N_{22}^{-1} N_{21} \\
L_{11}-L_{12} L_{22}^{-1} L_{21} & 0
\end{array}\right] .
\end{aligned}
$$


Then, by Lemma 2, we obtain

$$
E_{\alpha}\left(t^{\alpha} \mathcal{S}_{\Psi}\right)=\left[\begin{array}{cc}
E_{2 \alpha}\left(t^{2 \alpha} \mathcal{S}_{N} \mathcal{S}_{L}\right) & t^{\alpha} E_{2 \alpha, \alpha+1}\left(t^{2 \alpha} \mathcal{S}_{N} \mathcal{S}_{L}\right) \mathcal{S}_{N} \\
t^{\alpha} E_{2 \alpha, \alpha+1}\left(t^{2 \alpha} \mathcal{S}_{L} \mathcal{S}_{N}\right) \mathcal{S}_{L} & E_{2 \alpha}\left(t^{2 \alpha} \mathcal{S}_{L} \mathcal{S}_{N}\right)
\end{array}\right]
$$

It follows from Theorem 1 that

$$
\begin{aligned}
{\left[\begin{array}{l}
x_{1}(t) \\
y_{1}(t)
\end{array}\right] } & =z_{1}(t)=E_{\alpha}\left(\mathcal{S}_{\Psi} t^{\alpha}\right) z_{1}(0) \\
& =\left[\begin{array}{cc}
E_{2 \alpha}\left(t^{2 \alpha} \mathcal{S}_{N} \mathcal{S}_{L}\right) & t^{\alpha} E_{2 \alpha, \alpha+1}\left(t^{2 \alpha} \mathcal{S}_{N} \mathcal{S}_{L}\right) \mathcal{S}_{N} \\
t^{\alpha} E_{2 \alpha, \alpha+1}\left(t^{2 \alpha} \mathcal{S}_{L} \mathcal{S}_{N}\right) \mathcal{S}_{L} & E_{2 \alpha}\left(t^{2 \alpha} \mathcal{S}_{L} \mathcal{S}_{N}\right)
\end{array}\right]\left[\begin{array}{l}
x_{1}(0) \\
y_{1}(0)
\end{array}\right]
\end{aligned}
$$

Thus,

$$
\begin{aligned}
& x_{1}(t)=E_{2 \alpha}\left(t^{2 \alpha} \mathcal{S}_{N} \mathcal{S}_{L}\right) x_{1}(0)+t^{\alpha} E_{2 \alpha, \alpha+1}\left(t^{2 \alpha} \mathcal{S}_{N} \mathcal{S}_{L}\right) \mathcal{S}_{N} y_{1}(0) \\
& y_{1}(t)=t^{\alpha} E_{2 \alpha, \alpha+1}\left(t^{2 \alpha} \mathcal{S}_{L} \mathcal{S}_{N}\right) \mathcal{S}_{L} x_{1}(0)+E_{2 \alpha}\left(t^{2 \alpha} \mathcal{S}_{L} \mathcal{S}_{N}\right) y_{1}(0)
\end{aligned}
$$

Theorem 1 also implies that

$$
\left[\begin{array}{l}
x_{2}(t) \\
y_{2}(t)
\end{array}\right]=z_{2}(t)=-\Psi_{22}^{-1} \Psi_{21} z_{1}(t)=-\left[\begin{array}{cc}
0 & L_{22}^{-1} \\
N_{22}^{-1} & 0
\end{array}\right]\left[\begin{array}{cc}
0 & N_{21} \\
L_{21} & 0
\end{array}\right]\left[\begin{array}{l}
x_{1}(t) \\
y_{1}(t)
\end{array}\right]
$$

Hence, $x_{2}(t)=-L_{22}^{-1} L_{21} x_{1}(t)$ and $y_{2}(t)=-N_{22}^{-1} N_{21} y_{1}(t)$.

Corollary 3. From the notations and the assumption in Corollary 2, assume further that $L=N$. Then, the general solution of the system when $\alpha=1$ is given by Formula (21) where

$$
\begin{array}{ll}
x_{1}(t)=\cosh \left(t \mathcal{S}_{L}\right) x_{1}(0)+\sinh \left(t \mathcal{S}_{L}\right) y_{1}(0), & x_{2}(t)=-L_{22}^{-1} L_{21} x_{1}(t) \\
y_{1}(t)=\sinh \left(t \mathcal{S}_{L}\right) x_{1}(0)+\cosh \left(t \mathcal{S}_{L}\right) x_{1}(0), & y_{2}(t)=-N_{22}^{-1} N_{21} y_{1}(t) .
\end{array}
$$

Proof. We use the fact that $E_{2}(Z)=\cosh Z$ and $Z E_{2,2}\left(Z^{2}\right)=\sinh Z$ for any square matrix $Z$.

\subsection{Single Equations}

Corollary 4. Let $0<\alpha \leq 1$. Assume the notations and Assumption 1 in Section 3 for which $C_{i}=0, E_{i}=0$, so that $N=L=0, N_{i j}=L_{i j}=0$ for each $i, j \in\{1,2\}$, and the invertibility of $\Psi_{22}$ is reduced to the invertibility of both $M_{22}$ and $T_{22}$. Then, the general solution of the system:

$$
\sum_{i=1}^{k_{1}} P_{i} X^{(\alpha)}(t) R_{i}=\sum_{i=1}^{k_{2}} A_{i} X(t) B_{i}+U(t)
$$

is given by $X(t)=\operatorname{Vec}^{-1}\left(J_{2}\left[\begin{array}{l}x_{1}(t) \\ x_{2}(t)\end{array}\right]\right)$ where

$$
\begin{aligned}
& x_{1}(t)=E_{\alpha}\left(t^{\alpha} \mathcal{S}_{M}\right) x_{1}(0)+\int_{0}^{t}(t-\tau)^{\alpha-1} E_{\alpha}\left((t-\tau)^{\alpha} \mathcal{S}_{M}\right)(\Gamma \operatorname{Vec} U(\tau)) d \tau, \\
& x_{2}(t)=-M_{22}^{-1} M_{21} x_{1}(t)-M_{22}^{-1} J_{21} \operatorname{Vec} U(t),
\end{aligned}
$$

$\mathcal{S}_{M}=M_{11}-M_{12} M_{22}^{-1} M_{21}$ and $\Gamma=J_{11}-M_{12} M_{22}^{-1} J_{21}$. For the homogeneous case $U(t)=0$ of the system, we have $x_{1}(t)=E_{\alpha}\left(t^{\alpha} \mathcal{S}_{M}\right) x_{1}(0)$ and $x_{2}(t)=-M_{22}^{-1} M_{21} x_{1}(t)$. 
Proof. From the notations and the assumption, we have

$$
\begin{aligned}
\mathcal{S}_{\Psi} & =\left[\begin{array}{cc}
M_{11} & 0 \\
0 & T_{11}
\end{array}\right]-\left[\begin{array}{cc}
M_{12} & 0 \\
0 & T_{12}
\end{array}\right]\left[\begin{array}{cc}
M_{22}^{-1} & 0 \\
0 & T_{22}^{-1}
\end{array}\right]\left[\begin{array}{cc}
M_{21} & 0 \\
0 & T_{21}
\end{array}\right] \\
& =\left[\begin{array}{cc}
M_{11}-M_{12} M_{22}^{-1} M_{21} & T_{11}-T_{12} T_{22}^{-1} T_{21}
\end{array}\right], \\
0 & {\left[\begin{array}{cc}
J_{11} & 0 \\
0 & K_{11}
\end{array}\right]-\left[\begin{array}{cc}
M_{12} & 0 \\
0 & T_{12}
\end{array}\right]\left[\begin{array}{cc}
M_{22}^{-1} & 0 \\
0 & T_{22}^{-1}
\end{array}\right]\left[\begin{array}{cc}
J_{21} & 0 \\
0 & K_{21}
\end{array}\right] } \\
& =\left[\begin{array}{cc}
J_{11}-M_{12} M_{22}^{-1} J_{21} & 0 \\
0 & K_{11}-T_{12} T_{22}^{-1} K_{21}
\end{array}\right] .
\end{aligned}
$$

It follows from Theorem 1 and Lemma 2 that

$$
\begin{aligned}
{\left[\begin{array}{l}
x_{1}(t) \\
y_{1}(t)
\end{array}\right]=} & z_{1}(t)=E_{\alpha}\left(t^{\alpha} \mathcal{S}_{\Psi}\right) Z_{1}(0)+\int_{0}^{t}(t-\tau)^{\alpha-1} E_{\alpha}\left((t-\tau)^{\alpha} \mathcal{S}_{\Psi}\right)(\Gamma w(\tau)) d \tau \\
= & E_{\alpha}\left(t^{\alpha}\left[\begin{array}{cc}
M_{11}-M_{12} M_{22}^{-1} M_{21} & 0 \\
0 & T_{11}-T_{12} T_{22}^{-1} T_{21}
\end{array}\right]\right)\left[\begin{array}{l}
x_{1}(0) \\
y_{1}(0)
\end{array}\right] \\
& +\int_{0}^{t}(t-\tau)^{\alpha-1} E_{\alpha}\left((t-\tau)^{\alpha}\left[\begin{array}{cc}
M_{11}-M_{12} M_{22}^{-1} M_{21} & 0 \\
0 & T_{11}-T_{12} T_{22}^{-1} T_{21}
\end{array}\right]\right) \\
& \left(\left[\begin{array}{cc}
J_{11}-M_{12} M_{22}^{-1} J_{21} & K_{11}-T_{12} T_{22}^{-1} K_{21}
\end{array}\right]\left[\begin{array}{cc}
\operatorname{Vec} U(\tau) \\
0 & \operatorname{Vec} V(\tau)
\end{array}\right]\right) d \tau \\
= & {\left[\begin{array}{cc}
E_{\alpha}\left(t^{\alpha}\left(M_{11}-M_{12} M_{22}^{-1} M_{21}\right)\right) x_{1}(0) \\
0 & E_{\alpha}\left(t^{\alpha}\left(T_{11}-T_{12} T_{22}^{-1} T_{21}\right)\right) y_{1}(0)
\end{array}\right] } \\
& +\int_{0}^{t}(t-\tau)^{\alpha-1}\left[\begin{array}{r}
E_{\alpha}\left((t-\tau)^{\alpha}\left(M_{11}-M_{12} M_{22}^{-1} M_{21}\right)\right) \\
0
\end{array}\right. \\
& {\left[\begin{array}{ll}
\left(J_{11}-M_{12} M_{22}^{-1} J_{21}\right) \operatorname{Vec} U(\tau) \\
\left(K_{11}-T_{12} T_{22}^{-1} K_{21}\right) \operatorname{Vec} V(\tau)
\end{array}\right] d \tau . }
\end{aligned}
$$

Thus, $x_{1}(t)=E_{\alpha}\left(t^{\alpha} \mathcal{S}_{M}\right) x_{1}(0)+\int_{0}^{t}(t-\tau)^{\alpha-1} E_{\alpha}\left((t-\tau)^{\alpha} \mathcal{S}_{M}\right)(\Gamma \operatorname{Vec} U(\tau)) d \tau$. Theorem 1 also implies that

$$
\begin{aligned}
{\left[\begin{array}{l}
x_{2}(t) \\
y_{2}(t)
\end{array}\right] } & =z_{2}(t)=-\Psi_{22}^{-1} \Psi_{21} z_{1}(t)-\Psi_{22}^{-1} W_{2} w(t) \\
& =-\left[\begin{array}{cc}
M_{22}^{-1} & 0 \\
0 & T_{22}^{-1}
\end{array}\right]\left[\begin{array}{cc}
M_{21} & 0 \\
0 & T_{21}
\end{array}\right]\left[\begin{array}{l}
x_{1}(t) \\
y_{1}(t)
\end{array}\right]-\left[\begin{array}{cc}
M_{22}^{-1} & 0 \\
0 & T_{22}^{-1}
\end{array}\right]\left[\begin{array}{cc}
J_{21} & 0 \\
0 & K_{21}
\end{array}\right]\left[\begin{array}{l}
\operatorname{Vec} U(t) \\
\operatorname{Vec} V(t)
\end{array}\right] \\
& =-\left[\begin{array}{c}
M_{22}^{-1} M_{21} x_{1}(t)+M_{22}^{-1} J_{21} \operatorname{Vec} U(t) \\
T_{22}^{-1} T_{21} y_{1}(t)+T_{22}^{-1} K_{21} \operatorname{Vec} V(t)
\end{array}\right] .
\end{aligned}
$$

Hence, $x_{2}(t)=-M_{22}^{-1} M_{21} x_{1}(t)-M_{22}^{-1} J_{21} \operatorname{Vec} U(t)$.

Remark 1. The singular matrix fractional descriptor system

$$
A Y^{(\alpha)}(t)=B Y(t)+C U(t)
$$


considered in [19] is a spacial case of Corollary 4 when all matrices are square, $k_{1}=k_{2}=1$, and $B_{1}=R_{1}=I$. In this case, we put $J_{11}=C_{11}, J_{21}=C_{21}$ and partition

$$
J_{1} C=\left[\begin{array}{l}
C_{11} \\
C_{21}
\end{array}\right]
$$

Remark 2. From Corollary 4, assume further that $\alpha=1$. Since $E_{1}(Z)=e^{Z}$ for any square matrix $Z$, we have

$$
x_{1}(t)=e^{t \mathcal{S}_{M}} x_{1}(0)+\int_{0}^{t} e^{(t-\tau) \mathcal{S}_{M}}(\Gamma \operatorname{Vec} U(\tau)) d \tau
$$

\section{Descriptor Vector Systems}

In this section, we extract certain linear continuous-time descriptor (vector) systems from the main system (7).

Corollary 5. Let $0<\alpha \leq 1$. Let $A, C, E, G, P, Q \in M_{p, p}$ be given constant matrices and $u(t), v(t) \in M_{p, 1}$ given vector functions. Consider

$$
\begin{aligned}
& P x^{(\alpha)}(t)=A x(t)+C y(t)+u(t), \\
& Q y^{(\alpha)}(t)=E x(t)+G y(t)+v(t),
\end{aligned}
$$

with unknown vector function $x(t), y(t) \in M_{p, 1}$. We apply the notation $z_{1}(t), z_{2}(t), w(t), \Psi_{21}, \Psi_{22}, \mathcal{S}_{\Psi}$ and $\Gamma$ according to Theorem 1. Suppose $0<\operatorname{rank} P=\operatorname{rank} Q<p$. Then, the general solution of the system (23) is given by $x(t)=\Phi_{1}\left(z_{1}(t), z_{2}(t)\right)$ and $y(t)=\Phi_{2}\left(z_{1}(t), z_{2}(t)\right)$, where

$$
\begin{aligned}
& z_{1}(t)=E_{\alpha}\left(t^{\alpha} \mathcal{S}_{\Psi}\right) z_{1}(0)+\int_{0}^{t}(t-\tau)^{\alpha-1} E_{\alpha}\left((t-\tau)^{\alpha} \mathcal{S}_{\Psi}\right)(\Gamma w(\tau)) d \tau \\
& z_{2}(t)=-\Psi_{22}^{-1} \Psi_{21} z_{1}(t)-\Psi_{22}^{-1} W_{2} w(t) .
\end{aligned}
$$

Proof. We consider Theorem 1 in the particular case that $n=r=s=1, m=p=q, k_{i}=1$ and $R_{i}=S_{i}=B_{i}=D_{i}=F_{i}=H_{i}=$ [1] for all $i$. Then, system (7) is reduced to system (23). According to Theorem 1, we have $P=J, Q=K, A=M, C=N, E=L, G=T$ For each $i, j=1,2$, denote $A_{i j}=M_{i j}$, $C_{i j}=N_{i j}, E_{i j}=L_{i j}, G_{i j}=T_{i j}$. Now, the desired result follows from Theorem 1.

Corollary 6. Let $0<\alpha \leq 1$. Assume $P, A \in M_{p, p}$ be given constant matrices. Let $u(t) \in M_{p, 1}$ be a given vector function. Suppose $0<\operatorname{rank} P<p$. Then, the general solution of the system:

$$
P x^{(\alpha)}(t)=A x(t)+u(t)
$$

with unknown vector function $x(t) \in M_{p, 1}$ is given by

$$
x(t)=J_{2}\left[\begin{array}{l}
x_{1}(t) \\
x_{2}(t)
\end{array}\right],
$$

where

$$
\begin{aligned}
& x_{1}(t)=E_{\alpha}\left(t^{\alpha} \mathcal{S}_{A}\right) x_{1}(0)+\int_{0}^{t}(t-\tau)^{\alpha-1} E_{\alpha}\left((t-\tau)^{\alpha} \mathcal{S}_{A}\right)(\Gamma u(\tau)) d \tau, \\
& x_{2}(t)=-A_{22}^{-1} A_{21} x_{1}(t)-A_{22}^{-1} J_{21} u(t) .
\end{aligned}
$$

$\mathcal{S}_{A}=A_{11}-A_{12} A_{22}^{-1} A_{21}$ and $\Gamma=J_{11}-A_{12} A_{22}^{-1} J_{21}$. Here, the notations $x_{1}(t), x_{2}(t), A_{21}, A_{22}$ and $J_{21}$ are according to Corollary 5. 
Proof. From Corollary 5 putting $C_{i j}=0, E_{i j}=0, G_{i j}=I$ for all $i, j \in\{1,2\}$, we have

$$
\begin{aligned}
\mathcal{S}_{\Psi} & =\left[\begin{array}{cc}
A_{11} & 0 \\
0 & I
\end{array}\right]-\left[\begin{array}{cc}
A_{12} & 0 \\
0 & I
\end{array}\right]\left[\begin{array}{cc}
A_{22}^{-1} & 0 \\
0 & I
\end{array}\right]\left[\begin{array}{cc}
A_{21} & 0 \\
0 & I
\end{array}\right] \\
& =\left[\begin{array}{cc}
A_{11}-A_{12} A_{22}^{-1} A_{21} & 0 \\
0 & 0
\end{array}\right]
\end{aligned}
$$

and

$$
\begin{aligned}
\Gamma & =\left[\begin{array}{cc}
J_{11} & 0 \\
0 & K_{11}
\end{array}\right]-\left[\begin{array}{cc}
A_{12} & 0 \\
0 & I
\end{array}\right]\left[\begin{array}{cc}
A_{22}^{-1} & 0 \\
0 & I
\end{array}\right]\left[\begin{array}{cc}
J_{21} & 0 \\
0 & K_{21}
\end{array}\right] \\
& =\left[\begin{array}{cc}
J_{11}-A_{12} A_{22}^{-1} J_{21} & 0 \\
0 & K_{11}-K_{21}
\end{array}\right] .
\end{aligned}
$$

It follows Corollary 5 that

$$
\begin{aligned}
{\left[\begin{array}{l}
x_{1}(t) \\
y_{1}(t)
\end{array}\right]=} & z_{1}(t)=E_{\alpha}\left(t^{\alpha} \mathcal{S}_{\Psi}\right) z_{1}(0)+\int_{0}^{t}(t-\tau)^{\alpha-1} E_{\alpha}\left((t-\tau)^{\alpha} \mathcal{S}_{\Psi}\right)(\Gamma w(\tau)) d \tau \\
= & E_{\alpha}\left(t^{\alpha}\left[\begin{array}{cr}
A_{11}-A_{12} A_{22}^{-1} A_{21} & 0 \\
0 & 0
\end{array}\right]\right)\left[\begin{array}{l}
x_{1}(0) \\
y_{1}(0)
\end{array}\right] \\
& +\int_{0}^{t}(t-\tau)^{\alpha-1} E_{\alpha}\left((t-\tau)^{\alpha}\left[\begin{array}{cc}
A_{11}-A_{12} A_{22}^{-1} A_{21} & 0 \\
0 & 0
\end{array}\right]\right) \\
& \left(\left[\begin{array}{cc}
J_{11}-A_{12} A_{22}^{-1} J_{21} & 0 \\
0 & K_{11}-K_{21}
\end{array}\right]\left[\begin{array}{l}
u(\tau) \\
v(\tau)
\end{array}\right]\right) d \tau .
\end{aligned}
$$

Thus, $x_{1}(t)=E_{\alpha}\left(t^{\alpha} \mathcal{S}_{A}\right) x_{1}(0)+\int_{0}^{t}(t-\tau)^{\alpha-1} E_{\alpha}\left((t-\tau)^{\alpha} \mathcal{S}_{A}\right)(\Gamma u(\tau)) d \tau$. Corollary 5 also implies that

$$
\begin{aligned}
{\left[\begin{array}{l}
x_{2}(t) \\
y_{2}(t)
\end{array}\right] } & =z_{2}(t)=-\Psi_{22}^{-1} \Psi_{21} z_{1}(t)-\Psi_{22}^{-1} W_{2} w(t) \\
& =-\left[\begin{array}{cc}
A_{22}^{-1} & 0 \\
0 & I
\end{array}\right]\left[\begin{array}{cr}
A_{21} & 0 \\
0 & I
\end{array}\right]\left[\begin{array}{l}
x_{1}(t) \\
y_{1}(t)
\end{array}\right]-\left[\begin{array}{cc}
A_{22}^{-1} & 0 \\
0 & I
\end{array}\right]\left[\begin{array}{cc}
J_{21} & 0 \\
0 & K_{21}
\end{array}\right]\left[\begin{array}{l}
u(t) \\
v(t)
\end{array}\right]
\end{aligned}
$$

Hence, $x_{2}(t)=-A_{22}^{-1} A_{21} x_{1}(t)-A_{22}^{-1} J_{21} u(t)$.

The next corollary provides an alternative way to find the general solution of system (1) mentioned in the Introduction.

Corollary 7. Consider the linear continuous-time descriptor system (1) for which $E \in M_{n, n}$ is a singular matrix such that $0<l:=\operatorname{rank} E<n$. We put $E$ in a canonical form and make matrix/vector partitions for which there are invertible matrices $J_{1}, J_{2}$ such that

$$
J_{1} E J_{2}=\left[\begin{array}{ll}
I_{l} & 0 \\
0 & 0
\end{array}\right], \quad J_{1} A J_{2}=\left[\begin{array}{ll}
A_{11} & A_{12} \\
A_{21} & A_{22}
\end{array}\right], \quad J_{1} B=\left[\begin{array}{l}
B_{11} \\
B_{21}
\end{array}\right], \quad x(t)=J_{2}\left[\begin{array}{l}
x_{1}(t) \\
x_{2}(t)
\end{array}\right] .
$$


Suppose that $A_{22}$ is invertible matrix. Denote $\mathcal{S}_{A}=A_{11}-A_{12} A_{22}^{-1} A_{21}$ and $\Gamma=B_{11}-A_{12} A_{22}^{-1} B_{21}$. Then,

$$
\begin{aligned}
& x_{1}(t)=e^{t \mathcal{S}_{A}} x_{1}(0)+\int_{0}^{t} e^{(t-\tau) \mathcal{S}_{A}} \Gamma u(\tau) d \tau, \\
& x_{2}(t)=-A_{22}^{-1} A_{21} x_{1}(t)-A_{22}^{-1} B_{21} u(t) .
\end{aligned}
$$

Proof. From the first equation of (7), we apply a similar process as in the proof of Theorem 1 to get

$$
\begin{aligned}
x^{\prime}(t) & =A_{11} x_{1}(t)+A_{12} x_{2}(t)+B_{11} u(t), \\
0 & =A_{21} x_{1}(t)+A_{22} x_{2}(t)+B_{21} u(t) .
\end{aligned}
$$

Since $A_{22}$ is invertible, we can use Lemma 3 (with $\alpha=1$ ) to obtain $x_{1}(t)$ and $x_{2}(t)$.

\section{Conclusions}

We investigate a descriptor system of coupled generalized Sylvester matrix fractional differential equations in both non-homogeneous and homogeneous cases. All fractional derivatives considered here are taken in Caputo's sense. We explain a 4-step procedure to solve the descriptor system. Steps 1 and 4 are about transforming between matrices and vectors, and they require the vector operator and the Kronecker product. Step 2 is a coordinate transformation from original (vector) variables to new ones, and it is accomplished by matrix partitioning concerning a matrix canonical form concerning ranks. Step 3 relies on fractional differential equations. An explicit form of its general solution is thus given in terms of Mittag-Leffler functions. The main system includes certain systems of coupled matrix/vector differential equations and single matrix differential equations as special cases. In particular, we obtain an alternative procedure to solve linear continuous-time descriptor systems via a matrix canonical form. Our results include the previous works $[13,15,18-21]$ as special cases.

Author Contributions: All authors contributed equally and significantly in writing this article. All authors have read and agreed to the published version of the manuscript.

Funding: The first author received a financial support from the RA-TA graduate scholarship from the Faculty of Science, King Mongkut's Institute of Technology Ladkrabang, Grant No. RA/TA-2562-M-002 during her Master's study.

Acknowledgments: This work was supported by King Mongkut's Institute of Technology Ladkrabang.

Conflicts of Interest: The authors declare no conflict of interest.

\section{References}

1. Zhang, L.; Huang, B.; Lam, J. LMI synthesis of $H_{2}$ and mixed $H_{2} / H_{\infty}$ controllers for singular systems. IEEE Trans. Autom. Control 2003, AC-50, 615-626.

2. Brenan, K.E.; Campbell, S.; Petzold, L.R. Numerical Solution of Initial Value Problems in Differential Algebraic Equations; SIAM: Philadelphia, PA, USA, 1995.

3. Zhang, Q.L.; Lam, J.; Zhang, L.Q. Generalized Lyapunov equation for analyzing the stability of descriptor systems. In Proceedings of the 14th World Congress of IFAC, Beijing, China, 5-9 July 1999; pp. 19-24.

4. Men, B.; Zhang, Q.; Li, X .; Yang, C.; Chen, Y. The stability of linear descriptor systems. Internat J. Inform. Syst. Sci. 2006, 2, 362-374.

5. Campell, L.S.; Petzold, L.R. Canonical forms and solvable singular systems of differential equations. SIAM J. Algebraic Discret. Methods 1983, 4, 517-521. [CrossRef]

6. Dai, L. Singular Control Systems. In Lecture Notes in Control and Information Sciences; Springer: Berlin, Germany, 1989.

7. Gerdin, M. Parameter Estimation in Linear Descriptor Systems. Licentiate Thesis, Linköping University, Linköping, Sweden, 2004.

8. Bender, D.J.; Laub, A.J. The linear-quadratic optimal regulator for descriptor systems. IEEE Trans. Autom. Control 1987, AC32, 672-688. [CrossRef] 
9. Su, X.; Zhi, Y. Sufficient conditions of asymptotic stability of the time-varying descriptor systems. Int. J. Eng. Math. 2013, 2013, 920851. [CrossRef]

10. Lewis, E. A survey of linear singular systems. Circuits Syst. Signal Process. 1986, 5, 3-36. [CrossRef]

11. Wei, K. Stabilization of linear time-varying state feedback control. IEEE Trans. Control 1994, 39, $22-32$.

12. Takaba, T.; Morihira, N.; Katayama, K. A generalized Lyapunov theorem for descriptor system. Syst. Control Lett. 1995, 24, 49-51. [CrossRef]

13. Barnett, S. Matrix differential equations and Kronecker products. SIAM J. Appl. Math. 1973, 24, 1-5. [CrossRef]

14. Al Zhour, Z. Efficient solutions of coupled matrix and matrix differential equations. Intell. Control Autom. 2012, 3, 176-187. [CrossRef]

15. Kilicman, A.; Al Zhour, Z. The general common exact solutions of coupled linear matrix and matrix differential equations. J. Anal. Comput. 2005, 1, 15-30.

16. Al Zhour, Z. A computationally-efficient solutions of coupled matrix differential equations for diagonal unknown matrices. J. Math. Sci. Adv. Appl. 2008, 1, 373-387.

17. Al Zhour, Z. The general (vector) solutions of such linear (coupled) matrix fractional differential equations by using Kronecker structures. Appl. Math. Comput. 2014, 232, 498-510. [CrossRef]

18. Al Zhour, Z. New techniques for solving some matrix and matrix differential equations. Ain Shams Eng. J. 2015, 6, 347-354. [CrossRef]

19. Al Zhour, Z. The general solutions of singular and non-singular matrix fractional time-varying descriptor systems with constant coefficient matrices in Caputo sense. Alex. Eng. J. 2016, 55, 1675-1681. [CrossRef]

20. Saechai, S.; Chansangiam, P. Solving non-homogeneous coupled linear matrix differential equations in terms of matrix convolution product and Hadamard product. J. Inform. Math. Sci. 2018, 10, 237-245. [CrossRef]

21. Kongyaksee, R.; Chansangiam, P. Solving system of nonhomogeneous coupled linear matrix differential equations in terms of Mittag-Leffler matrix functions. J. Comput. Anal. Appl. 2019, 26, 1150-1160.

22. Saechai, S.; Chansangiam, P. General exact solution to a system of coupled linear matrix differential equations. Adv. Appl. Math. Sci. 2017, 16, 151-161.

23. Balanchan, K.; Kokila, J.; Trujillo, J.J. Relative controllability of fractional dynamical system with multiple delays in control. Comput. Math. Appl. 2012, 64, 3037-3045. [CrossRef]

24. Balanchan, K.; Kokila, J. On the controllability dynamic systems. Int. J. Appl. Math. Comput. Sci. 2012, 22, 523-531 [CrossRef]

25. Yousefi, F.; Rivaz, A.; Chen, W. The construction of operational matrix of fractional integration for solving fractional differential and integro-differential equations. Neural Comput. Appl. 2019, 31, 1867-1878. [CrossRef]

26. Horn, R.A.; Johnson, C.R. Topics in Matrix Analysis; Cambridge University Press: New York, NY, USA, 1991.

27. Sadeghi, A.; Cardoso, J.R. Some notes on properties of the matrix Mittag-Leffler function. Appl. Math. Comput. 2018, 338, 733-738. [CrossRef]

(C) 2020 by the authors. Licensee MDPI, Basel, Switzerland. This article is an open access article distributed under the terms and conditions of the Creative Commons Attribution (CC BY) license (http:/ / creativecommons.org/licenses/by/4.0/). 